\title{
INDICADORES TERRITORIALES: ¿DEGRADACIÓN DEL PATRIMONIO NATURAL TURÍSTICO EN LA REGIÓN DE LOS RÍOS?, PERIODO 1998-2007
}

\author{
Autores: \\ Gastón Vergara \\ Universidad de Austral de Chile \\ Facultad de Ciencias Económicas y Administrativas \\ Instituto de Estadística \\ Valdivia, Chile \\ gastonvergara@uach.cl \\ Víctor Sandoval \\ Universidad de Austral de Chile \\ Facultad de Ciencias Forestales \\ Instituto de Manejo \\ Valdivia, Chile \\ Juan Carlos Miranda \\ Universidad de Austral de Chile \\ Facultad de Ciencias Económicas y Administrativas \\ Instituto de Estadística \\ Valdivia, Chile
}

\section{RESUMEN}

La presente investigación presenta el cambio del recurso natural en la Región de Ríos durante el periodo 1998 y 2007, obteniéndose como resultado un conjunto de indicadores que determinan y explican el cambio patrimonial territorial.

El año 2007 se realizó un monitoreo de actualización de los recursos naturales de la Región de los Ríos, tomando como información base los datos gráficos y alfanuméricos del proyecto Catastro Nacional de los Recursos Naturales de Chile del año 1998. Con los dos periodos de información se realizaron cruces de datos que permitieron explicar espacialmente lo que está ocurriendo en la Región, cuantificandose el cambio con la construcción de matrices e indicadores territoriales del periodo.

Los resultados permitieron detectar un incremento considerable de las plantaciones, es decir, la introducción de especies exóticas a la Región, lo que ha significado la disminución del bosque nativo, matorrales, terrenos agrícolas y causes de agua, tipos de recursos naturales esenciales para el desarrollo turístico.

Las principales conclusiones del trabajo se relacionan con que el territorio de la Región de los Ríos ha experimentado cambios significativos en sus recursos naturales en el periodo estudiado 1998 - 2007; La Región de los Ríos presenta un ordenamiento territorial deficiente y ambiguo, no existiendo por un lado instrumentos objetivos de planificación y por otro conflictos de intereses entre el desarrollo turístico e industrial; es urgente e indispensable de parte de las autoridades disponer de indicadores que permitan realizar una real planificación de los recursos naturales de la Región.

Palabras clave: Monitoreo, Indicadores territoriales, Recurso natural. 
TERRITORIAL INDICATORS: DEGRADATION OF THE TOURIST NATURAL PATRIMONY IN REGION DE LOS RIOS?, 1998-2007 PERIOD.

\author{
Authors: \\ Gastón Vergara \\ Universidad de Austral de Chile \\ Facultad de Ciencias Económicas y Administrativas \\ Instituto de Estadística \\ Valdivia, Chile \\ gastonvergara@uach.cl \\ Víctor Sandoval \\ Universidad de Austral de Chile \\ Facultad de Ciencias Forestales \\ Instituto de Manejo \\ Laboratorio de Geomática \\ Valdivia, Chile

\section{Juan Carlos Miranda} \\ Universidad de Austral de Chile \\ Facultad de Ciencias Económicas y Administrativas \\ Instituto de Estadística \\ Valdivia, Chile
}

\title{
ABSTRACT
}

This report presents the change of the natural resource in Region de los Rios during 1998 - 2007, which results in a set of indicators that determines and explains the territorial patrimonial change. In 2007, a monitoring to update the natural resources information of Region de Los Rios was carried out, taking as base information the graphic and alphanumeric data obtained through National Registry of Chile's Natural Resources from year 1998. Both information periods were crisscrossed which allowed explaining what is happening space-wise in the Region, also change through the building of territorial matrixes and indicators was quantified.

The results helped detecting a considerable increase of plantations, i.e. introduction of exotic species to the Region; on one hand, the introduction of foreign species meant a decrease of the native forest, bushes, agricultural lands, and water courses, which are considered essential natural resources to tourism development. The growth of urban and industrial areas has also meant the decrease of wetlands (26,1 ha), agricultural lands (48,9 ha), prairies and bushes (814,3 ha).

The report main conclusions are related to the fact that Region de Los Rios territory has undergone significant changes in its natural resources during the studied period (19982007); Region de Los Rios presents a deficient and ambiguous territorial planning, on one hand by the lacking of planning objective instruments and, on the other hand, conflict of interests between the tourist and industrial developments; it is urgent and indispensable, on the part of the authorities, that they have indicators that will help perform an actual planning of the Region's natural resources.

Key words: Monitoring, territorial indicators, natural resource 


\section{INTRODUCCIÓN}

La Región de los Ríos se crea a partir del año 2007, con la promulgación de la ley $N^{\circ} 20.174$ por parte de la Presidenta de la República, Michelle Bachelet, en Valdivia el 16 de Marzo de 2007, publicándose definitivamente en el diario oficial el 5 de Abril de 2007. La composición geográfica corresponde a la separación territorial que antiguamente pertenecía a la Región de los Lagos. La Región de los Ríos está dividida administrativamente en dos provincias y doce comunas, las cuales corresponden a: Valdivia (Capital Regional), Corral, La Unión, Río Bueno, Lago Ranco, Futrono, Panguipulli, Lanco, Mariquina, Máfil, Los Lagos y Paillaco (ver figura ${ }^{\circ} 1$ ).

La Región de los Ríos tiene una superficie de 18.429,5 km2, lo que representa el 2,45\% de la superficie del país, ocupada principalmente por terrenos con Plantaciones artificiales, Bosque nativo y Bosque mixto, alcanzando el 56.7\% de la superficie total (Instituto Forestal de Chile ); las área urbanas e industriales constituyen solamente el $0.25 \%$ del total.

Según cifras entregadas por el último censo de población (2002), la población es de 356.396 habitantes, equivalente al $2.36 \%$ de la población nacional, alcanzando un porcentaje de ruralidad del 31,7\% y una densidad poblacional de 19,3 habitantes por kilómetro cuadrado (Instituto Nacional de Estadisticas).

Figura $N^{\circ} 1$. Mapa de la Región de Los Ríos

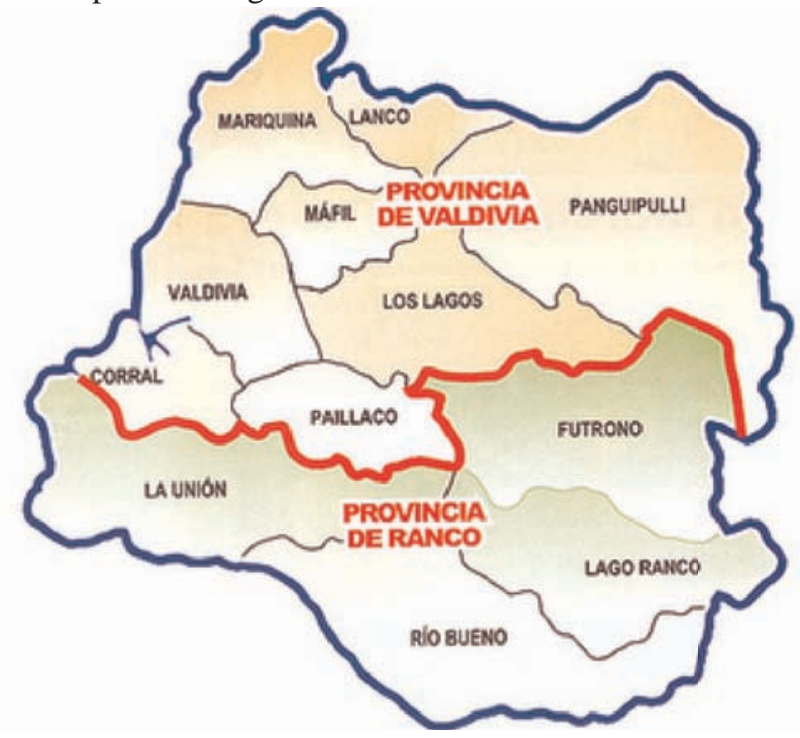

Fuente: Intendencia Regional 
Las nuevas autoridades deben asumir la administración territorial y sus demandas, para lo cual cuentan con un presupuesto de 39 mil millones, asociado al presupuesto, es común leer declaraciones de las principales autoridades como las siguientes

"Hoy día tenemos un presupuesto sin precedentes, que genera equidad territorial, y una tremenda oportunidad, porque tenemos un presupuesto que proporcionalmente al tamaño territorial y a la población es el más alto de todas las regiones del país, pero también nos genera un tremendo desafio de poder ser eficientes y eficaces en efectivamente transformar estas ideas y estos proyectos en obras u acciones" (Intendencia Región de Los Ríos).

Los consejeros regionales comentan la forma en que la comisión de hacienda del Core, toma sus decisiones frente a las diferentes inversiones en la nueva Región.

“... por último el convencimiento de los consejeros, que la propuesta que en definitiva se va aprobar efectivamente responde a las necesidades de cada comuna y los proyectos provinciales y regionales, representando oportunidades ciertas de potenciar sectores económicos relevantes para nuestra región, como también apoyar iniciativas sociales y culturales que deben ayudar a hacer de esta región un región distinta" (Intendencia Región de Los Ríos).

La falta de indicadores se detectó en forma dramática durante el proceso de formulación y gestión de la Región de los Ríos el año 2007, donde el Comité Técnico para la Nueva Región Provincia de Valdivia debía formular propuestas a la autoridad gubernamental y política, que le permitieran sustentar la nueva figura geopolítica (Miranda B, Miranda J). Las necesidades de información e indicadores que permitieran defender muchas demandas hacia la nueva Región se reflejaban, por una parte el no disponer de la información requerida y por otra, las múltiples fuentes dispersas con los datos originales, situación que hacia impracticable la recolección y el procesamiento de los mismos, que les permitiera a los constituyentes del comité, construir indicadores debidamente validados y sustentables.

Dada la importancia de una buena administración en la Región de Los Ríos, como una región naciente y modelo, siendo una de las que ha despertado el mayor interés mundial, frente a conflictos en el ámbito ambiental (Corporación Nacional Forestal), por decisiones mal informadas, adquiere una gran trascendencia el disponer de indicadores que provengan de fuentes confiables, únicas y confeccionados con técnicas que los conviertan en información inteligente para el apoyo de las autoridades en la toma de sus decisiones, en los diferentes ámbitos que la región requiere. 
El objetivo del presente trabajo, consiste en la presentación del diseño e implementación de un Sistema de Información Geográfico (Sandoval et al) que permita monitorear los recursos naturales de la Región de los Ríos, diseñar y construir indicadores que revelen y expliquen los cambios ocurridos en los Recursos Naturales y sus implicancias futuras.

\section{MATERIAL Y MÉTODO}

Para el diseño y construcción del SIG-REGIONAL, se efectúo en una primera fase, un análisis intensivo de los datos gráficos y alfanuméricos provenientes del Proyecto Catastro y Monitoreo. Las herramientas de análisis y descripción de datos utilizados en esta etapa, correspondieron a las metodologías normalmente aplicados a este tipo de procesos, como son los diagramas de flujo de datos y los diagramas de entidad relación. Posteriormente se identificaron las necesidades de información propias de la Región, que debieran dar cumplimiento con los objetivos estratégicos referidos a la temática territorial, y finalmente se determinaron los requerimientos cartográficos y alfanumérico, necesarios para responder las diferentes consultas técnicas y la construcción de indicadores. En la figura $\mathrm{n}^{\circ} 2$ se presenta un flujo de las principales actividades realizadas durante la elaboración de la cartografía base y enlace con las actividades del proyecto monitoreo. 
Figura $\mathrm{N}^{\circ} 2$. Flujo de información del Sistema de Información Geográfico

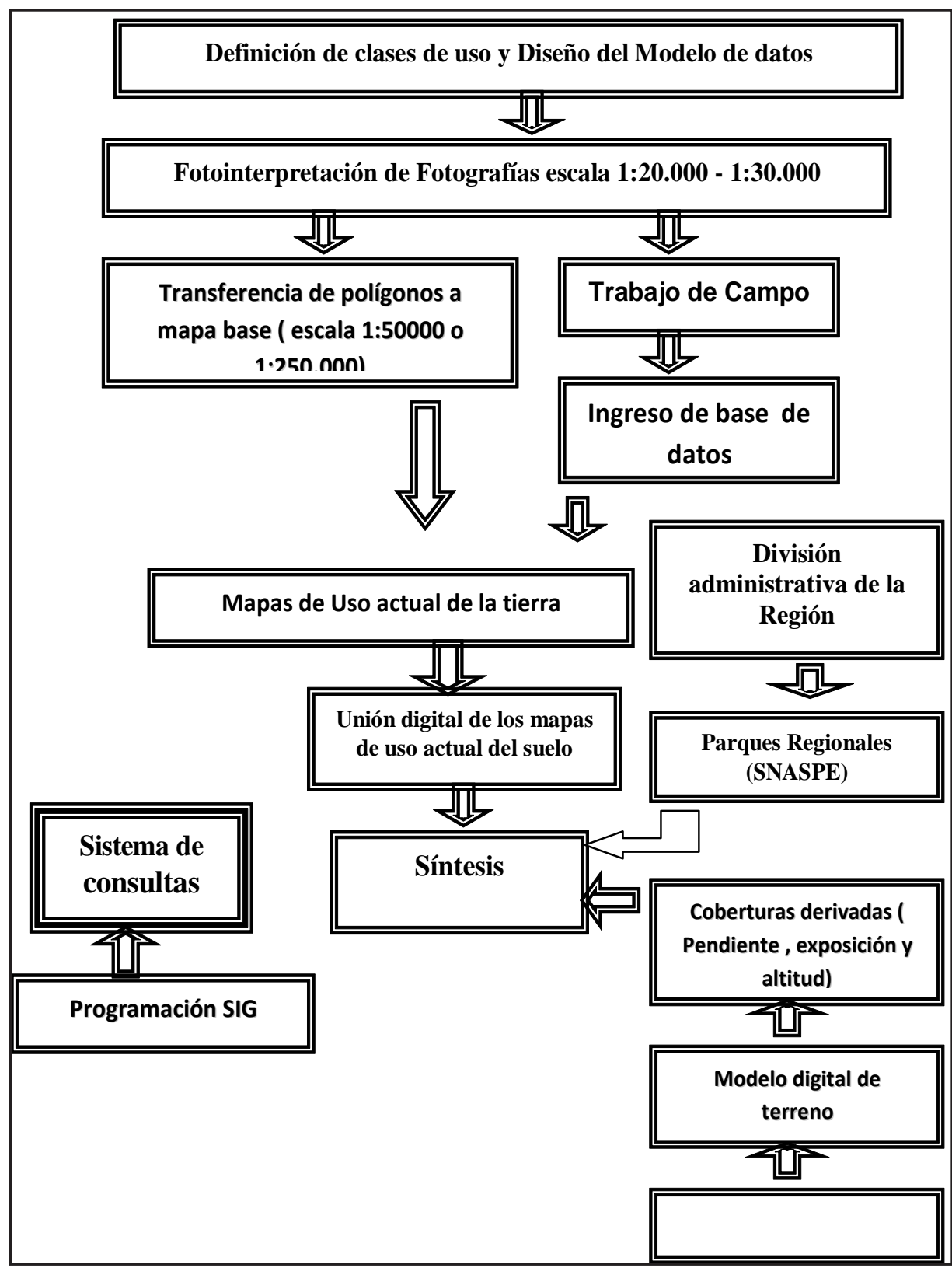


Otra fase importante del sistema consiste en la construcción de un módulo de procesamiento y validación de la información gráfica y alfanumérica que formarán parte integral del sistema, en la figura $\mathrm{n}^{\circ} 3$ se pueden visualizar las etapas constituyentes de los procesos gráficos y alfanuméricos, desde la creación de coberturas, unión y/o intersección de las distintas coberturas temáticas. Participando en forma paralela el procesamiento de la información alfanumérica proveniente de los formularios de campo.

Figura $N^{\circ} 3$. Diagrama proceso de información gráfica y alfanumérica.

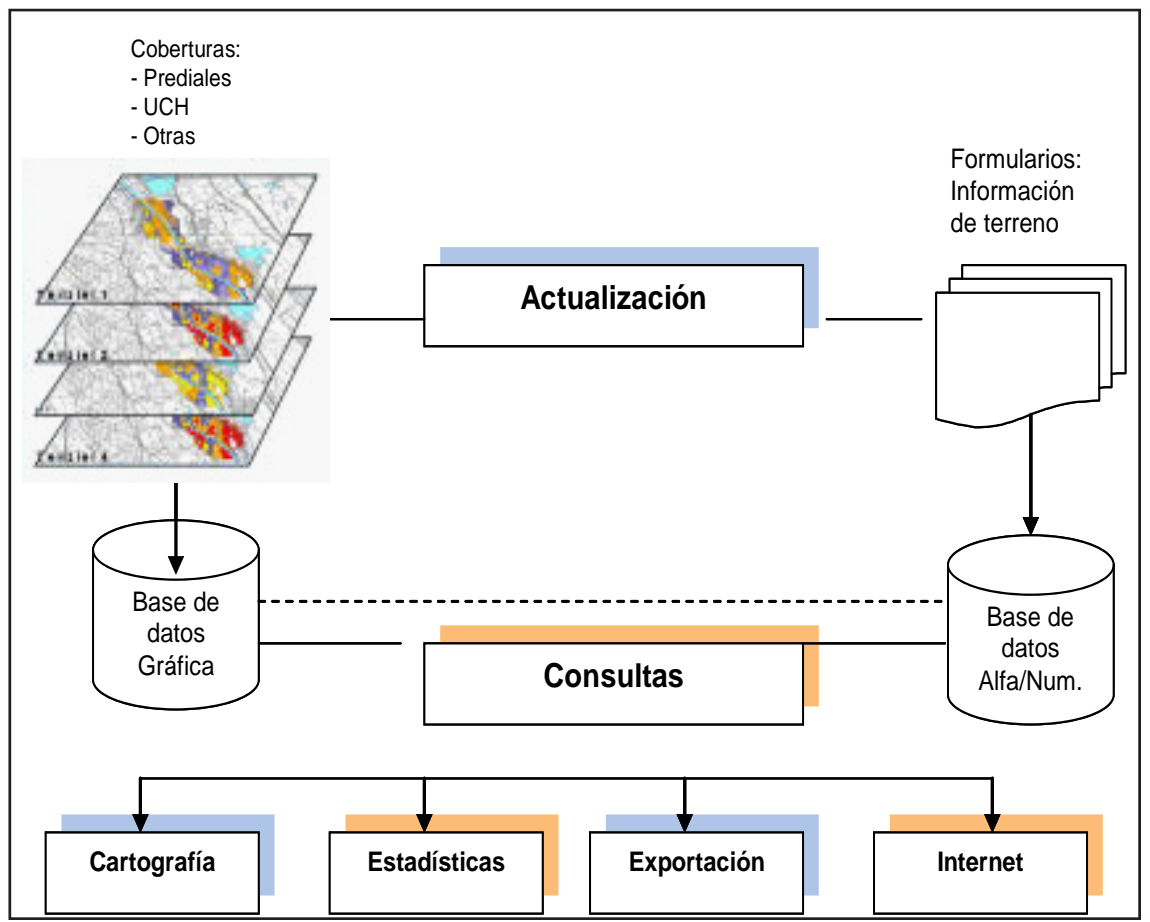

Sobre los Indicadores Regionales en la Región de los Ríos (IRR). En primer lugar se necesario recordar que un indicador es una información procesada, generalmente de carácter cuantitativo, que genera una idea clara y accesible sobre un fenómeno complejo, su evolución, y sobre cuánto difiere de una situación deseada (Blanco. H et al). A nivel nacional tenemos algunos indicadores económicos de uso permanente, como son los casos del producto interno bruto, la inflación y el desempleo, permitiendo evaluar el desempeño de la economía. 
Las características que cumplirán los Indicadores Regionales (IRR), están relacionados con los siguientes puntos:

a) El conjunto de indicadores deben representar una mirada de futuro, por ejemplo al mostrar el valor de los indicadores a través de los años, o al incluir metas o valores límites.

b) Los indicadores deben considerar la distribución equitativa de las condiciones entre la comunidad. Al respecto, los indicadores pueden reflejar aspectos distributivos dentro de una comunidad o a través de un área geográfica.

c) Los indicadores deberían distinguir el origen y el destino de acciones sociales, económicas y ambientales.

Si bien es cierto que el origen de la información para la generación de los IRR, está radicada en un Data Warehouse, la génesis en sí debe estar basada en el cumplimiento de una serie de etapas que se detallan a continuación.

a) Conocer a los actores regionales relevantes, las iniciativas relacionadas (plan estratégico de desarrollo de la región), y las instancias de planificación disponibles (como la estrategia de desarrollo regional).

b) Desarrollar un taller regional con representantes de los sectores público, privado y de la sociedad civil, intentando identificar y acordar las principales prioridades y objetivos para el desarrollo regional. Los resultados de este taller, junto a la información recabada en la región, nos permiten elaborar un perfil regional, el cual incluye características generales; antecedentes sobre las dimensiones económica, social, y una síntesis de las prioridades para el desarrollo de la región desagregadas en objetivos específicos.

c) Utilizar el perfil regional para crear una primera propuesta de indicadores.

d) La propuesta preliminar de indicadores es discutida y perfeccionada con la ayuda de algunos especialistas regionales.

e) Finalmente la propuesta de indicadores es presentada y discutida en un nuevo taller con la participación de los principales actores regionales

A continuación se presentan dos ejemplos esquemáticos del proceso desarrollado en la elaboración de IRR. 
Cuadro $\mathrm{N}^{\circ} 1$. Caracterización indicador turístico

\begin{tabular}{|c|c|c|}
\hline Prioridad & $\begin{array}{l}\text { Impulsar el desarrollo económico } \\
\text { de la Región }\end{array}$ & \multirow{2}{*}{$\begin{array}{l}\text { Corresponde al resultado de } \\
\text { la primera y segunda etapa: } \\
\text { Recopilación de información, } \\
\text { identificación de actores, y primer } \\
\text { taller para elaborar perfil regional. }\end{array}$} \\
\hline Objetivo & $\begin{array}{l}\text { Impulsar la competitividad de la } \\
\text { región en el desarrollo de servicios } \\
\text { de estadía turística }\end{array}$ & \\
\hline \multicolumn{3}{|l|}{ Indicador } \\
\hline Nombre & Servicios turísticos integrales & \multirow{3}{*}{$\begin{array}{l}\text { Corresponde al resultado de la } \\
\text { segunda etapa: Propuestapreliminar } \\
\text { de indicadores, discusión con } \\
\text { actores regionales seleccionados, } \\
\text { refinamiento de la propuesta, } \\
\text { presentación en taller regional. }\end{array}$} \\
\hline Fórmula & $\begin{array}{l}\left(\mathrm{N}^{\circ} \text { de turistas que se hospedan en }\right. \\
\text { Valdivia / } \mathrm{N}^{\circ} \text { de turistas que solo } \\
\text { pasan por valdivia }) * 100\end{array}$ & \\
\hline $\begin{array}{l}\text { Pertinencia para } \\
\text { el desarrollo de } \\
\text { región }\end{array}$ & $\begin{array}{l}\text { Económico } \\
\text { La detención de turistas en Valdivia } \\
\text { podría significar ingresos relevantes } \\
\text { por servicios turísticos. } \\
\text { Institucional } \\
\text { Se requiere por una parte, potenciar } \\
\text { los servicios turísticos, de manera } \\
\text { de impulsar la competitividad de la } \\
\text { región, por otro lado es necesario } \\
\text { realizar tareas de promoción, que } \\
\text { pasa por la coordinación entre } \\
\text { actores públicos v privados. }\end{array}$ & \\
\hline
\end{tabular}


Cuadro $N^{\circ}$ 2.Caracterización indicador de ocupación territorial

\begin{tabular}{|c|c|c|}
\hline Prioridad & $\begin{array}{l}\text { Impulsar el desarrollo económico de } \\
\text { la Región }\end{array}$ & \multirow{2}{*}{$\begin{array}{l}\text { Corresponde al resultado de } \\
\text { la primera y segunda etapa: } \\
\text { Recopilación de información, } \\
\text { identificación de actores, y primer } \\
\text { taller para elaborar perfil regional. }\end{array}$} \\
\hline Objetivo & $\begin{array}{l}\text { Impulsar la competitividad de la } \\
\text { región en el uso de sus recursos } \\
\text { naturales. }\end{array}$ & \\
\hline \multicolumn{3}{|l|}{ Indicador } \\
\hline Nombre & Movilidad en ocupación territorial & \multirow{3}{*}{$\begin{array}{l}\text { Corresponde al resultado de la } \\
\text { segunda etapa: Propuesta preliminar } \\
\text { de indicadores, discusión con } \\
\text { actores regionales seleccionados, } \\
\text { refinamiento de la propuesta, } \\
\text { presentación en taller regional. }\end{array}$} \\
\hline Fórmula & $\begin{array}{l}\text { ((Ha con tipo uso del suelo en año } \\
\text { base - Ha con tipo uso del suelo en } \\
\text { año actual) / Ha con tipo uso del } \\
\text { suelo en año actual) } * 100\end{array}$ & \\
\hline $\begin{array}{l}\text { Pertinencia } \\
\text { para el } \\
\text { desarrollo de } \\
\text { región }\end{array}$ & $\begin{array}{l}\text { Económico } \\
\text { La transformación de los recursos } \\
\text { naturales de la región puede } \\
\text { significar ingresos/pérdidas en el } \\
\text { cambio del uso del suelo. } \\
\text { Institucional } \\
\text { Se requiere por una parte, } \\
\text { potenciar el desarrollo de algunas } \\
\text { actividades en el uso del suelo } \\
\text { (incentivos), de manera de impulsar } \\
\text { la competitividad de la región, por } \\
\text { otro lado es necesario realizar tareas } \\
\text { de ordenamiento y legislación en la } \\
\text { protección de los recursos naturales. }\end{array}$ & \\
\hline
\end{tabular}

\section{RESULTADOS Y DISCUSION}

\section{Indicadores Territoriales.}

La información disponible y validada para presentar resultados discutibles a nivel Regional, corresponden a los datos entregados por el Laboratorio de Geomática de la Universidad Austral de Chile, organismo encargado en procesar la información territorial a nivel nacional desde el año 1994, fecha en la cual se confeccionó el catastro nacional.

Los resultados presentados en tablas y gráficos corresponden con cifras que a la fecha de obtener la información del monitoreo territorial de la región - Junio del 2007-, aún se encontraban en proceso de corrección, situación que podría variar con los 
resultados finales entregados posteriormente al organismo solicitante y propietario de la información Corporación Nacional Forestal de Chile CONAF.

Cuadro N³. Ocupación territorial y porcentaje de cambio periodos 1998 - 2007

\begin{tabular}{|l|c|r|r|r|}
\hline Usos Territorio 1998 & $\begin{array}{c}\text { Superficie } \\
\text { Corregida } \\
\text { (ha) } \mathbf{1 9 9 8}\end{array}$ & $\begin{array}{c}\text { Superficie } \\
\text { Actualizada } \\
\text { (ha) } 2007\end{array}$ & $\begin{array}{r}\text { Superficie de } \\
\text { Cambio (ha) }\end{array}$ & $\begin{array}{l}\text { \% de } \\
\text { Cambio }\end{array}$ \\
\hline 1. Áreas Urbanas e Industriales & 4784.6 & 5749.2 & 964.6 & 20.2 \\
\hline 2. Terrenos Agrícolas & 16585.8 & 16503.9 & -81.9 & -0.5 \\
\hline 3. Praderas y Matorrales & 584338.5 & 548300.1 & -36038.4 & -6.2 \\
\hline 4.1 Plantaciones & 162577.8 & 207178.6 & 44600.8 & 27.4 \\
\hline 4.2 Bosque Nativo & 868841.8 & 861991.9 & -6849.9 & -0.8 \\
\hline 4.3 Bosque Mixto & 12130.8 & 11686.1 & -444.7 & -3.7 \\
\hline 5. Humedales & 15299.1 & 15146 & -153.1 & -1.0 \\
\hline $\begin{array}{l}\text { 6. Áreas Desprovistas de } \\
\text { Vegetación }\end{array}$ & 55408.6 & & & -1967.6 \\
\hline 7. Nieves y Glaciares & 11192.9 & 11192.9 & & -3.6 \\
\hline 8. Cuerpos de Agua & 109147.8 & 109117.9 & -29.9 & 0.0 \\
\hline Total (ha) & $\mathbf{1 8 4 0 3 0 7 . 7}$ & $\mathbf{1 8 4 0 3 0 7 . 6}$ & & 0.0 \\
\hline
\end{tabular}

Fuente: Información obtenida del Sistema de Catastro y Monitoreo en el uso del suelo de Chile (CONAF, 2007)

Se puede apreciar en la columna Superficie de Cambio (ha) que el uso asociado a Áreas Urbanas e Industriales de la región, presentaron un crecimiento considerable (20.2\%), idéntica situación podemos observar con el uso de Plantaciones, las cuales crecieron en un 27.4\%, sin embargo el uso Praderas y Matorrales disminuyo en un 6.2\%, el uso Áreas sin vegetación también disminuyó en un 3.7\%, las causas de este fenómeno regional es justamente las que deben analizar expertos y autoridades, apreciaciones preliminares indican que la actual instalación de una planta de celulosa estaría incentivando el uso del suelo en plantaciones artificiales de pino radiata y eucaliptos.

\section{Análisis multidimensional del uso del suelo.}

Los datos fueron sometidos a un análisis multidimensional, con representaciones de los años o periodos de cambios, usos del territorio y superficie cubierta por el uso. 
Figura $\mathrm{N}^{\circ}$ 5. Análisis multidimensional de los datos en el Data Warehouse

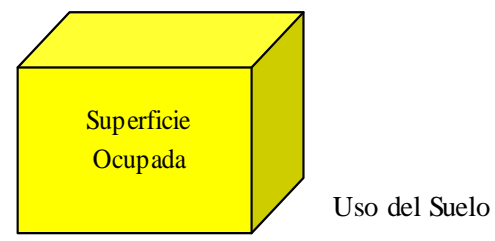

Periodo
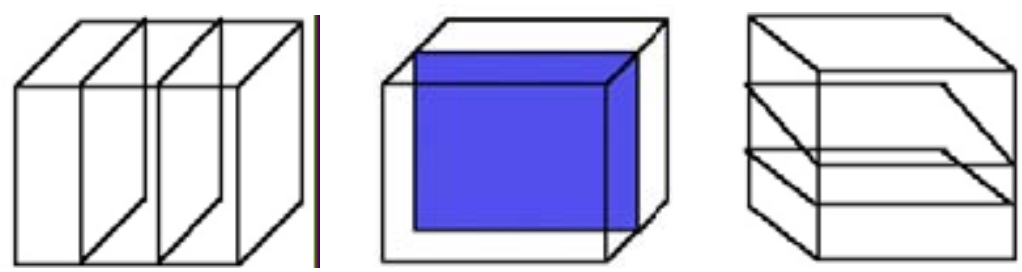

Matrices de cambio en el uso del territorio

Líneas de contorno

Se obtuvo una matriz de cambio del territorio regional, donde en el uso territorial por cada periodo, además se obtienen los aumentos o disminuciones de superficie por tipo de uso y cuáles de éstos llevaron al incremento o disminución del periodo analizado, según las existencias anteriores o actuales. 
Cuadro $\mathrm{N}^{\circ} 4$. Matriz de cambio con uso del territorio regional, periodos 1998 - 2007

\begin{tabular}{|c|c|c|c|c|c|c|c|c|c|c|c|c|c|c|}
\hline \multicolumn{2}{|c|}{ 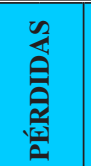 } & & $\stackrel{\text { N }}{\sim}$ & 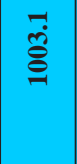 & 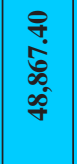 & $\begin{array}{l}\text { R } \\
\text { ச் }\end{array}$ & 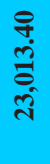 & $\begin{array}{l}\text { ते } \\
\text { లె } \\
\text {. }\end{array}$ & ชั๋ & $\begin{array}{l}\infty \\
\stackrel{\infty}{\infty} \\
\stackrel{\infty}{-1}\end{array}$ & 0 & $\begin{array}{l}\text { L } \\
\text { ஜூ }\end{array}$ & & $\begin{array}{l}0 \\
\text { 守 } \\
\text { N }\end{array}$ \\
\hline$\underset{\tilde{\theta}}{\tilde{\theta}}$ & 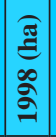 & & ָ̃ & 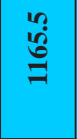 & $\begin{array}{l}\text {.े } \\
\text { ठै. } \\
\text { In }\end{array}$ & लृ & $\begin{array}{l}\text { के } \\
\text { ఫे } \\
\text { ฟิ }\end{array}$ & $\begin{array}{l}\text { ஸें } \\
\text { లై }\end{array}$ & 节 & $\begin{array}{l}\infty \\
\stackrel{\infty}{\infty} \\
\stackrel{\infty}{-1}\end{array}$ & 0 & 苋 & 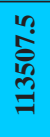 & \\
\hline \multirow{10}{*}{ 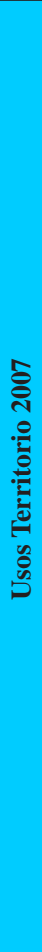 } & $\begin{array}{l}0 \\
\infty\end{array}$ & & 0 & 0 & 0 & 0 & 0 & 0 & 0 & 0 & 0 & 0 & 0 & 0 \\
\hline & $\stackrel{2}{\circ}$ & & 0 & 0 & 0 & 0 & 0 & 0 & 0 & 0 & 0 & 0 & 0 & 0 \\
\hline & $\begin{array}{l}0 \\
0\end{array}$ & & 0 & -1 & $\begin{array}{l}\varphi \\
\stackrel{\infty}{\sim}\end{array}$ & $\begin{array}{l}\overrightarrow{0} \\
\text { ஸे }\end{array}$ & $\stackrel{\text { Pִ }}{\rightarrow}$ & L & 0 & 0 & 0 & 0 & ஸ̊ & 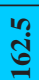 \\
\hline & iी & & 0 & 0 & 0 & 0 & 0 & 0 & 0 & 0 & 0 & 0 & 0 & 0 \\
\hline & \multirow{3}{*}{${ }_{i}$} & $m^{m}$ & 0 & 0 & 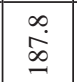 & તิ่ & $\stackrel{\infty}{\stackrel{\sim}{\sim}}$ & 0 & 0 & 0 & 0 & 0 & $\underset{\sim}{\vec{J}}$ & $\underset{\sim}{\vec{J}}$ \\
\hline & & f & 0 & ஜ் & 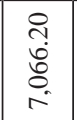 & $\hat{\hat{\theta}}$ & 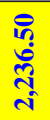 & $\frac{n}{6}$ & $\begin{array}{l}\infty \\
\text { L் }\end{array}$ & $\begin{array}{l}\varphi \\
\dot{q}\end{array}$ & 0 & $\vec{\lambda}$ & 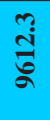 & 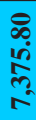 \\
\hline & & f & $\stackrel{\sim}{\forall}$ & $\begin{array}{l}\stackrel{+}{~} \\
\stackrel{\infty}{\infty}\end{array}$ & 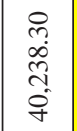 & $\begin{array}{l}\stackrel{0}{0} \\
\dot{0} \\
\stackrel{0}{0} \\
\dot{m}\end{array}$ & 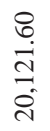 & 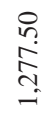 & $\begin{array}{l}\hat{o} \\
\stackrel{8}{f}\end{array}$ & $\begin{array}{l}8 \\
\stackrel{-}{-1} \\
=\end{array}$ & 0 & $\begin{array}{l}9 \\
\text { ம் }\end{array}$ & $\begin{array}{l}\text { में } \\
\text { बूं } \\
\text { हू }\end{array}$ & 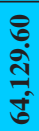 \\
\hline & ले & & 0 & $\hat{\ddot{g}}$ & $\begin{array}{l}\text { Nิ } \\
\text { ભి } \\
\text { ஸे } \\
\text { N }\end{array}$ & $\begin{array}{l}\dot{\varphi} \\
\dot{\mathscr{~}} \\
\end{array}$ & 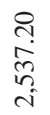 & $\stackrel{9}{\stackrel{9}{-}}$ & $\begin{array}{l}\infty \\
\stackrel{\nu}{ }\end{array}$ & $\underset{N}{\stackrel{J}{N}}$ & 0 & $\stackrel{\text { مִ }}{\stackrel{2}{2}}$ & 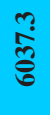 & 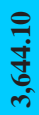 \\
\hline & $\stackrel{0}{i}$ & & 0 & ชี & $\begin{array}{l}\text { Ñ } \\
\text { Hี่ }\end{array}$ & กิ & 令 & 0 & 0 & 0 & 0 & 0 & $\begin{array}{l}\text { ષ્ } \\
\stackrel{0}{\circ}\end{array}$ & สิ \\
\hline & $\stackrel{\circ}{=}$ & & ஜั & $\begin{array}{l}\text { の } \\
\text { oీ }\end{array}$ & $\underset{\infty}{\stackrel{M}{+}}$ & $\stackrel{m}{\sim}$ & $\stackrel{\varphi}{\mathscr{r}}$ & $\stackrel{\sim}{\sim}$ & $\ddot{\mathscr{v}}$ & 0 & 0 & 0 & $\stackrel{10}{=}$ & \&े \\
\hline \multicolumn{3}{|c|}{ 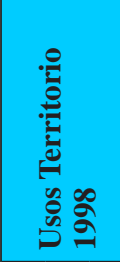 } & 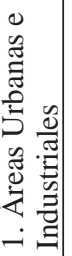 & 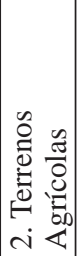 & 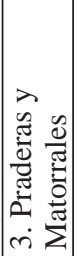 & 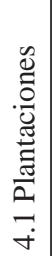 & 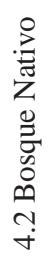 & 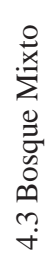 & 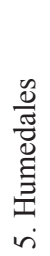 & 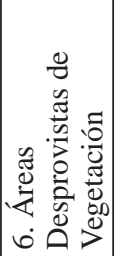 & 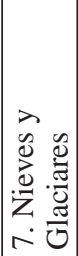 & 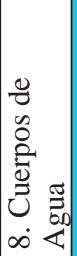 & 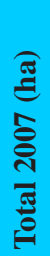 & 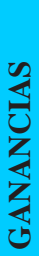 \\
\hline
\end{tabular}

Fuente: Información obtenida del Catastro y Monitoreo en el uso del suelo de Chile (CONAF, 2007) 
Si analizamos la matriz con las direcciones de cambio territorial, podemos observar que los principales cambios en el incremento de las Áreas Urbanas (20.2\%), provienen de la transformación de praderas y matorrales e incluyendo parte de los humedales (26.1 ha).

Al analizar el origen de de la superficie territorial que se está destinado al uso de Plantaciones artificiales, el análisis muestra que su principal origen corresponde a Praderas y Matorrales (40238.3 ha) que se están destinando a este nuevo tipo de actividad, en un segundo lugar con 20121.60 ha corresponden a Bosque nativo, recurso que es de vital importancia para el visitante turístico en el sur de Chile.

\section{BIBLIOGRAFÍA}

- Corporación Nacional Forestal, (2007), Monitoreo Nacional del Uso del Suelo en Chile, CONAF, Santiago, Chile.

- Instituto Nacional de Estadísticas, (2002), Censo de Población y Vivienda en Chile 2002, INE, Santiago, Chile.

- Intendencia Región de Los Ríos (2007) Unidad de Comunicaciones, , , Valdivia, Chile.

- Miranda B. y Miranda, J. ,(2007), La evaluación de políticas públicas: algunas precisiones conceptuales. Edita Universidad Austral de Chile.

- Corporación Nacional Forestal, (2007), Plan Integral de Gestión Ambiental del Humedal de Río Cruces, CONAF, Santiago, Chile.

- Blanco, Hernán, Wautiez, Françoise, Llavero, Angel et al., (2001) Indicadores regionales de desarrollo sustentable en Chile: ¿Hasta qué punto son útiles y necesarios?, EURE, Santiago, Chile.

- Sandoval V., Real P., Vergara G. y Trincado G. (2002), Desarrollo de un Sistema de Información Geográfico Forestal en Chile, Bosque, Valdivia, Chile.

Recibido: $15 / 06 / 2010$

Aprobado:25/10/2010

Arbitrado anonimamente 